\title{
A TORELLI THEOREM FOR SIMPLY CONNECTED ELLIPTIC SURFACES WITH A SECTION AND $p_{g} \geqslant 2$
}

BY K. CHAKIRIS

0. The purpose of this note is to announce some recent results on the period mapping for simply connected elliptic surfaces with a section and $p_{g} \geqslant 2$. Among other things, we are able to prove the period mapping for such surfaces has degree one, that is to say, it is generically injective. In the terminology of [C, G], this is called the weak global Torelli theorem. In order to make a precise statement of our results, we must introduce some notation.

1. Let $V$ be a surface with at most rational double points, $\bar{V} \stackrel{\sigma}{\rightarrow} V$ its minimal resolution; let $\psi: V \rightarrow \mathbf{P}_{1}$ be a proper analytic map, whose generic fibre is a smooth elliptic curve. Let $C_{u}=\psi^{-1}(u), \bar{\psi}=\psi \circ \sigma$, and $\bar{C}_{u}=\bar{\psi}^{-1}(u)$. Assume $\exists s \exists s: \mathbf{P}_{1} \longrightarrow V$ is a section of $V \stackrel{\psi}{\rightarrow} \mathbf{P}_{1}$, and that $V$ is smooth along $s\left(\mathbf{P}_{1}\right)$; let $\bar{s}$ be the corresponding section of $\bar{V} \stackrel{\psi}{\rightarrow} \mathbf{P}_{1}$. Now assume $p_{g}(\bar{V})=$ $n \geqslant 1$, and $\bar{V}$ is a minimal surface; $\bar{s} \cdot \bar{C}_{u}=1, \bar{s}^{2}=-(n+1), \bar{C}_{u}^{2}=0 . V \stackrel{\psi}{\rightarrow}$ $\mathbf{P}_{1}$ is called an elliptic fibration with a section (see $[\mathbf{K}]$ ). We also require that $\sigma(D)=$ a point for all irreducible curves $D \subset \bar{V}$, with $\bar{s} \cdot D=0=\bar{C}_{u} \cdot D$.

Let $H^{2}(\bar{V} ; \mathbf{Z})_{0}=\left(\mathbf{Z}_{c_{1}}\left(\bar{C}_{u}\right)+\mathbf{Z} \cdot c_{1}(s)\right)^{\perp}$, where $c_{1}(D)$ denotes the 1 st Chern class of a divisor; this is an orthogonal direct summand of $H^{2}(\bar{V} ; \mathbf{Z})$ and hence must be a unimodular lattice. It is uniquely characterized by the integer $n$; fix a copy and call it $H$; set $\Lambda=H \oplus(\mathrm{Z} c+\mathrm{Z} s)$, where $c \cdot H=s \cdot H=0, c^{2}=0$, $s \cdot c=1, s^{2}=-(n+1)$.

Let $\varphi: H^{2}(\bar{V} ; \mathbf{Z}) \rightarrow \Lambda$ be an isomorphism of unimodular lattices $\varphi\left(c_{1}\left(\bar{C}_{u}\right)\right)$ $=c, \varphi\left(c_{1}(s)\right)=s, \varphi\left(c_{1}\left(K_{\bar{V}}\right)\right)=(n-1) \cdot c$ where $K_{\bar{V}}$ is the canonical divisor, and hence $\varphi\left(H^{2}(\bar{V} ; \mathrm{Z})_{0}\right)=H$. Following [P-S, Săf], we will call $\varphi$ a marking, and the pair $(V, \varphi)$ a marked surface of type $(H, \Lambda, c, s)$. Let $L_{V} \subset H^{2}(\bar{V} ; \mathrm{Z})_{0}$ be the euclidean sublattice generated by all elements of the form $c_{1}(D), D>0$, $\sigma(D)=$ a point; and let $\widetilde{H}_{V}=\left(L_{V}\right)^{\perp} ; L_{V}$ is negative definite. For any euclidean lattice $E$, we let $O(E)$ be the orthogonal group of $E .^{1}$ This is a linear algebraic group defined over $\mathbf{Q}$; let $O(E)_{\mathbf{Z}}=\left\{g \in O(E)_{\mathbf{R}} \mid g E=E\right\}$. For any $\alpha \in E, \alpha^{2}=-2$, let

Received by the editors November 10, 1981.

1980 Mathematics Subject Classification. Primary 32G20.

1 The pairing on $E$ may be indefinite. 
$S_{\alpha} \in O(E)_{Z}$ be the reflection defined by $S_{\alpha}(\beta)=\beta+(\alpha \cdot \beta) \alpha, \forall \beta \in E$; and let $\Re(E) \subset O(E)_{\mathbf{Z}}$ be the subgroup generated by the reflections.

Let $\left(V_{i}, \varphi_{i}\right), i=1,2$, be two marked surfaces, $f: V_{1} \rightarrow V_{2}$ an isomorphism, $\bar{f}$ its unique lift to $\bar{V}_{1} \rightarrow \bar{V}_{2}$. Assume that $\exists g \in \Re(L), \exists \varphi_{1} \circ \bar{f}^{*}=$ $\bar{g} \circ \varphi_{2}$, where $\bar{g}$ is the obvious extension of $g$ to $\Lambda$; then we say that the marked surfaces are equivalent, $\left(V_{1}, \varphi_{1}\right) \sim\left(V_{2}, \varphi_{2}\right)$.

Let $J=J(H, \Lambda, c, s)$ be the set of marked surfaces of type $(H, \Lambda, c, s)$, modulo this equivalence. Let $\Gamma=O(H)_{\mathrm{Z}}$, we define an action of $\Gamma$ on $J$ by: $\gamma \cdot(V, \varphi)=(V, \bar{\gamma} \circ \varphi)$, where $\gamma \in \Gamma$, and $\bar{\gamma}$ is its extension to $\Lambda$, defined by $\bar{\gamma}(c)=c, \bar{\gamma}(s)=s$.

It can be shown that $J$ is a smooth analytic space, $\Gamma$ acts properly discontinuously on $J$, and $J / \Gamma=M$ is irreducible. ${ }^{2}$ Moreover, if $n \geqslant 2$ then for marked surfaces $\left(V_{i}, \varphi_{i}\right), i=1,2: \exists f, V_{1} \stackrel{f}{\rightarrow} V_{2}, \exists f$ is a birational isomorphism with $f\left(s_{1}\right)=s_{2}$ iff $\exists \gamma \in \Gamma$, such that $\gamma \cdot\left(V_{1}, \varphi_{1}\right) \sim\left(V_{2}, \varphi_{2}\right)$.

2. Let $\operatorname{Gr}\left(n, H_{\mathrm{C}}\right)$ be the Grassmannian of $n$-planes in $H_{\mathrm{C}} ; \xi \in \mathrm{Gr}\left(n, H_{\mathrm{C}}\right)$ corresponding to the $n$-plane $F(\xi) \subset H_{\mathrm{C}}$. Following Griffiths [G], let $\check{D}(H)=\{\xi \in$ $\left.\operatorname{Gr}\left(n, H_{\mathbf{C}}\right) \mid u \cdot v=0, \forall u, v \in F(\xi)\right\} ;$ let $D(H)=\{\xi \in \check{D}(H) \mid \bar{u} \cdot u>0, \forall u \in$ $F(\xi)-\{0\}\} . D(H)$ is an open subset of $\check{D}(H)$, homogeneous under the action of $O(H)_{\mathrm{R}} ; \Gamma$ acts properly discontinuously on $D(H)$. Let $z=(V, \varphi) \in J$ be a marked surface; and $H^{2,0}(V) \subset H^{2}(\bar{V}, \mathrm{C})$ be the subspace corresponding to holomorphic $(2,0)$ forms. Let $\varphi: H^{2}(\bar{V}, \mathrm{C}) \rightarrow \Lambda_{\mathrm{C}}$ also denote the extension of $\varphi$ to $H^{2}(\bar{V}, \mathrm{C})$; clearly $\varphi\left(H^{2,0}(V)\right) \subset H_{\mathrm{C}}$, since $\varphi^{-1}(c), \varphi^{-1}(s)$ both correspond to algebraic cycles. Define $\Phi(z)=\xi \in D(H)$ by $\varphi\left(H^{2,0}(V)\right)=F(\xi)$; clearly $\Phi(\gamma \cdot z)$ $=\boldsymbol{\gamma} \cdot \Phi(z)$.

By fundamental results of Griffiths, $\Phi$ is holomorphic. Let us assume $n \geqslant 2$.

MAIN TheOREM. There is a $\Gamma$-invariant dense open subset of $J$ on which $\Phi$ is injective.

REMARK. This is equivalent to a "degree one" statement.

Following the philosophy in [P-S, Săf], the essential idea is to reduce the problem to a "special" sublocus of $J$.

3. Let $(V, \varphi) \in J$, assume $n \geqslant 2$, then the elliptic fibration $\psi: V \rightarrow \mathbf{P}_{1}$ is uniquely determined. Let $C_{a_{1}}, \ldots, C_{a_{r}}$ be the singular fibres of $\psi$, so that $C_{u}$ is smooth $\forall u \in \mathbf{P}_{1}^{\prime}=\mathbf{P}_{1}-\left\{a_{1}, \ldots, a_{r}\right\}$; choose $a \in \mathbf{P}_{1}^{\prime}$, and $e_{1}, e_{2} \in H_{1}\left(C_{a}, \mathbf{Z}\right)$, $e_{1} \cdot e_{2}=1$. Let $\tau_{i}$ be a local parameter at $a_{i}$, the loop $\gamma:[0,1] \rightarrow \mathbf{P}_{1}^{\prime}$ defined by $\tau_{i}(\gamma(t))=\epsilon \exp \{2 \pi i t\}$ defines a conjugacy class in $\pi_{1}\left(\mathbf{P}_{1}^{\prime}, a\right)$, any member of

2 It follows from the work of Kas that $M$ is irreducible. 
which will be referred to as a positive loop about $a_{i}$. Let $T: \pi_{1}\left(\mathbf{P}_{1}^{\prime}, a\right) \rightarrow$ $\operatorname{SL}(2, Z)$ be the monodromy of $V \rightarrow P_{1}$, corresponding to the choice of $a$, and $e_{1}, e_{2}$. For $w \in \operatorname{SL}(2, \mathrm{Z})$, we let $[w]$ denote its conjugacy class; $C_{a_{i}}$ is said to be of type $[w]$ if: $T(\gamma)=[w]$ for any positive loop $\gamma$ about $a_{i}$ (see [M]).

We will call $(V, \varphi)$ a special elliptic surface if $T\left(\pi_{1}\left(\mathbf{P}_{1}^{\prime}, a\right)\right) \subset\left\{ \pm 1_{2}\right\}, 1_{2}=$ $\left(\begin{array}{l}10 \\ 0\end{array}\right)$. Let $J_{1}=\{z \in J \mid z=(V, \varphi)$ is a special elliptic surface $\}$. One can show that $J_{1}$ is a smooth analytic subvariety of $J$, invariant under $\Gamma$, and $J_{1} / \Gamma$ is irreducible. These surfaces will play a role similar to the one played by special Kummer surfaces in [P-S, Săf] with one major exception: they will not be a dense subset.

4. Let $\Phi_{1}: J / \Gamma \rightarrow D / \Gamma$ be the map induced by $J \stackrel{\Phi}{\rightarrow} D, D=D(H)$, $\pi: D \rightarrow D / \Gamma$ the qt. map. Assume there is a compact projective variety $Y$, divisors, $E^{\prime}, E^{\prime \prime} \subset Y$, a proper surjective map $g: Y-E^{\prime}-E^{\prime \prime} \rightarrow M=J / \Gamma$; the map $f=\Phi_{1} \circ g$ extends to a proper holomorphic map $f_{1}, f_{1}: Y-E^{\prime \prime} \rightarrow D / \Gamma$. Moreover, assume $f^{-1}\left(J_{1} / \Gamma\right)$ is quasi-projective. This will all hold in our situation, due to the construction of $M$ as a quotient of an open subset of a suitable Hilbert scheme; together with the results of [G]. Let $Z \subset J_{1}$ be an irreducible component, then we may write

$$
\overline{\Phi(J) ~ \supset \Phi(J) ~ \supset ~} \overline{\Phi(J)}-N, \quad \overline{\Phi(Z)} \supset \Phi(Z) \supset \overline{\Phi(Z)}-N_{1}
$$

where $\overline{\Phi(\mathcal{J})}, \overline{\Phi(Z)}, N, N_{1}$ are all closed analytic subvarieties of $D$ (see [G]).

Consider the following statements:

(a) $(d \Phi)_{z}$ is injective at each $z \in Z$,

(b) $\Phi^{-1}(\Phi(Z)) \subseteq \bigcup_{\gamma \in \Gamma} \gamma \cdot Z$,

(c) $\Phi / Z: Z \rightarrow \Phi(Z)$ is injective,

(d) $\{\gamma \in \Gamma \mid \gamma(Z)=Z\}=\{\gamma \in \Gamma \mid \gamma \overline{\Phi(Z)} \cap \overline{\Phi(Z)}=\overline{\Phi(Z)}\}$,

(e) $\Phi(Z) \Phi \pi^{-1}\left(f_{1}\left(E^{\prime}-E^{\prime \prime}\right)\right)$.

The following is not difficult to prove:

Lемма. (a)-(e) imply the main theorem.

Let us make some remarks concerning the proofs of (a)-(d).

(a) This may be proven by using the techniques of [Kii].

(b) This is proven by the same methods used by [P-S, Saf] to characterize special Kummer surfaces, by means of their lattice of algebraic cycles.

(c) and (d) are reduced to the Torelli theorem for hyperelliptic curves, by means of the results contained in [B]. The idea of using the results in [B] may also be found in [P-S, Săf], however we use them somewhat differently. In order to understand our method of dealing with statement (e), we need to introduce some definitions. 
By a degeneration of elliptic fibrations with section we will mean the following:

(i) connected analytic spaces $V, D, \operatorname{dim}_{C} V=3, \operatorname{dim}_{C} D=2$, proper maps, $\Psi, \rho, p$, making the following diagram commute.

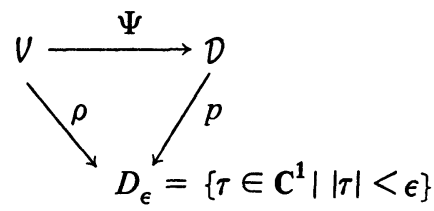

Set $V_{t}=\rho^{-1}(t), \Delta_{t}=p^{-1}(t), \Delta_{t}$ is assumed to be connected, $\forall t \neq 0$; the generic fibre of $\Psi$ is a smooth elliptic curve.

(ii) $V_{t}, \forall t \neq 0$, has at most rational double points; $D-\Delta_{0}$ is smooth.

(iii) There is a holomorphic map $s: D \rightarrow V$ with $\Psi \circ s=\mathrm{id}_{D}$, i.e. a section. We will restrict our attention to the case where $\Delta_{t} \cong \overline{\bar{P}}_{2}, \forall t \neq 0$. Let $P$ denote the double covering of the Hirzebruch surface $\Sigma_{2} \stackrel{P_{2}}{\rightarrow} \mathbf{P}_{1}$ branched along $B \in\left|3 S_{\infty}+S\right|$, where $S_{\infty}$ is the unique section of $p_{2}$ with $S_{\infty}^{2}=-2$, and $S \sim S_{\infty}$ $+2 F, F \sim p_{2}^{-1}(u)$; we require $S_{\infty}$ to be an isolated component of $B$, thereby occurring with multiplicity 3 . Let

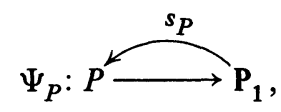

be the fibration induced by $p_{2}, s_{P}$ the section defined by $S \subset B$. Each fibre of $\Psi_{P}$ is a rational curve with a cusp, whose singularity has the form $z^{2}=y^{3} . P$, together with its fibration, will be called a connecting component. The degeneration of elliptic fibrations with section

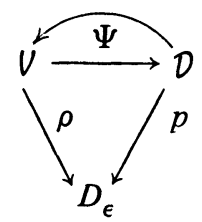

for which $V / D_{\epsilon}-\{0\}$ has trivial monodromy as a fibration of surfaces with rational double points, will be called stable if the following holds:

(1) $\Delta_{0}$ has only ordinary nodes, and $p$ vanishes to multiplicity one on each component of $\Delta_{0}$. Set $\Delta_{0}=\bigcup \Gamma_{i}$, each $\Gamma_{i} \cong \mathrm{P}_{1}$, and $X_{i}=\Psi^{-1}\left(\Gamma_{i}\right)$. Each $X_{i}$ occurs with multiplicity one.

(2) Each fibre of $\Psi$, when reduced, is an irreducible curve. Set $C_{u}=$ $\Psi^{-1}(u), \forall u \in D ; V$ is smooth along $s(D)$. $\Gamma_{j}=\varnothing$.

(3) If the generic fibres of $X_{i} \rightarrow \Gamma_{i}$ and $X_{j} \rightarrow \Gamma_{j}$ are smooth then $\Gamma_{i} \cap$ 
(4) If the generic fibre of $X_{i} \rightarrow \Gamma_{i}$ is smooth, $\overline{\Delta_{0}-\Gamma_{i}} \cap \Gamma_{i}=\left\{q_{1}, \ldots\right.$, $\left.q_{l}\right\}$, we let $\mu_{i}$ be the number of singular fibres of $\left.X_{i}\right|_{\Gamma_{i}}-\left\{q_{1}, \ldots, q_{l}\right\}$, properly counted. If the generic fibre of $X_{i} \rightarrow \Gamma_{i}$ is singular, set $\mu_{i}=0$. We require that $\Sigma_{j} \mu_{j}=12(n+1)$, where $p_{g}\left(V_{t_{0}}\right)=n, t_{0} \neq 0 .^{3}$

(5) If the generic fibre of $X_{i} \rightarrow \Gamma_{i}$ is smooth, and $p_{g}\left(X_{i}\right)=0$ then $\Gamma_{i}$ meets at least two other components of $\Delta_{0}$; if $\Gamma_{i}$ meets exactly two other components, then $\mu_{i}>0$.

(6) If the generic fibre of $X_{k} \rightarrow \Gamma_{k}$ is singular, then $X_{k}$ is a connecting component and $\Gamma_{k}$ meets exactly two other components of $\Delta_{0}, \Gamma_{k^{\prime}}, \Gamma_{k^{\prime \prime}}$. Set $\left\{q^{\prime}\right\}=\Gamma_{k^{\prime}} \cap \Gamma_{k},\left\{q^{\prime \prime}\right\}=\Gamma_{k^{\prime \prime}} \cap \Gamma_{k}$, then the generic fibres of $X_{k^{\prime}} \rightarrow \Gamma_{k^{\prime}}$, $X_{k^{\prime \prime}} \rightarrow \Gamma_{k^{\prime \prime}}$ are smooth; and let $C_{q^{\prime}}$ be of type $\left[w_{1}\right], C_{q^{\prime \prime}}$ of type $\left[w_{2}\right]$. Let $w_{i}=s_{i} u_{i}, i=1,2$ be their Jordan decomposition into semisimple and unipotent elements, $s_{i}$ semisimple, $u_{i}$ unipotent. Then, $u_{1} \neq 1$ iff $u_{2} \neq 1$; and $\left[s_{1}^{-1}\right]=$ $\left[s_{2}\right] \neq[1]$.

TheOREM (STABLE REDUCTION). Let $V$, D etc. be a degeneration of elliptic fibrations with section, such that $\left.V\right|_{D_{\epsilon}^{\prime}}, D_{\epsilon}^{\prime}=D_{\epsilon}-\{0\}$ has trivial monodromy as a fibration of surfaces with rational double points, then after a base extension $\varphi: D_{\epsilon_{1}} \rightarrow D_{\epsilon}, V \times{ }_{\varphi} D_{\epsilon_{1}}$ is $D_{\epsilon_{1}}$-birationally equivalent to a stable degneration.

This theorem is the key element in obtaining a proof of statement (e), above. To use it, we let the $Y$, used to dominate $M$, be obtained from a certain Hilbert scheme, $X \rightarrow Y$ the universal surface over $Y$, the fibres of $X \mid Y-E^{\prime}-E^{\prime \prime}$ being elliptic surfaces with at most rational double points $E^{\prime}-E^{\prime \prime}=E_{1} \cup \cdots \cup E_{m} \cup \widetilde{E}$, where $\left.X\right|_{E_{i}}$ has only fibres which fulfill properties (1)-(6) above, and $f_{1}(\widetilde{E}) \subset$ $\bigcup_{j} f_{1}\left(E_{j}\right)$. Due to properties (1)-(6), if we let $E$ be any one of the $E_{i}, \exists$ euclidean sublattices $H_{j} \subset H, 1 \leqslant j \leqslant r$ each $H_{j}$ having a nonzero even number of positive eigenvalues, $\left(H_{1}+\cdots+H_{r}\right)^{\perp}$ being negative definite, $H_{j} \subset H_{i}^{\perp} \forall i \neq j$; and a holomorphic map of $\widetilde{D} / \widetilde{\Gamma} \stackrel{\widetilde{F}}{\rightarrow} D / \Gamma$, where $\widetilde{D}=\Pi_{i} D\left(H_{i}\right), \widetilde{\Gamma}=\Pi O\left(H_{i}\right)_{\mathrm{Z}}, \widetilde{F}$ induced by the inclusion $\left(H_{1}+\cdots+H_{r}\right) \subset H$. Moreover, $\widetilde{F}(\widetilde{D} / \Gamma) \supset f_{1}(E)$.

Let $\Gamma_{Z}=\{\gamma \in \Gamma \mid \gamma(Z)=Z\} ; A_{\mathbf{R}}\left(\Gamma_{Z}\right)$ its Zariski closure in $O(H)_{\mathbf{R}}$; then $\Phi(Z) \subset \pi^{-1}\left(f_{1}(E)\right)$ implies that some conjugate of $A_{R}\left(\Gamma_{Z}\right)$ must leave an orthogonal decomposition $H_{\mathrm{R}}=H^{\prime}+H^{\prime \prime}$, invariant, each $H^{\prime}, H^{\prime \prime}$ having a nonzero number of positive eigenvalues. It may be verified that this is impossible.

\section{REFERENCES}

[C, G] J. Carlson and P. Griffiths, Infinitesimal variations of Hodge structure and the global Torelli problem (A. Beauville, ed.), Journées de Géométrie Algebrique d'Angers juillet 1979.

[Kii] K. I. Kĩ, The local Torelli theorem for varieties with divisible canonical class, Izv. Akad. Nauk 42 (1978). English transl. Math. USSR Izv. 12 (1978), No. 1.

${ }^{3}$ It follows from this, and condition (6), that $\Sigma_{i} p_{g}\left(X_{i}\right)=n$. 
[P-S, Să] I. I. Pyateckî̌-Shapiro and I. R. Safarevic, A Torelli theorem for algebraic surfaces of type K3, Izv. Akad. Nauk SSSR 35 (1971), No. 3. English transl. Math. USSR Izv. 5 (1971), No. 3.

[G] P. Griffiths, Periods of integrals on algebraic manifolds. III (Some global differential-geometric properties of the period mapping), Inst. Hautes Études Sci. Publ. Math. 38 (1970), 125-180.

[B] A. Borel, Density and maximality of arithmetic groups, J. Reine Angew. Math. 224 (1966), 78-89.

[K] K. Kodaira, On compact complex analytic surfaces. I, Ann. of Math. (2) 71 (1960), 111-152; On compact analytic surfaces. II, III, Ann. of Math. (2) 77 (1963), 563626; 78 (1963), $1-40$.

[M] B. Moishezon, Complex surfaces and connected sums of projective planes, Lecture Notes in Math., Vol. 603, Springer-Verlag, Berlin and New York, 1977.

DEPARTMENT OF MATHEMATICS, HARVARD UNIVERSITY, CAMBRIDGE, MASSACHUSETTS 02138 